\title{
Antibody-Mediated “Universal” Osteoclast Targeting Platform using Calcitonin as a Model Drug
}

\author{
Madhuri Newa $•$ Krishna Hari Bhandari • Lili Tang $•$ Rohit Kalvapalle $•$ Mavanur Suresh • Michael R. Doschak
}

Received: 17 September 2010 / Accepted: 19 January 2011 / Published online: 8 February 2011

(C) The Author(s) 2011. This article is published with open access at Springerlink.com

\begin{abstract}
Purpose To generate and characterize a specific monoclonal antibody (mAb) against recombinant human RANK receptor and to develop an antiresorptive strategy using this $\mathrm{mAb}$ as an osteoclast-targeting platform that selectively targets osteoclast cells whilst delivering an attached (i.e. chemically conjugated) active drug cargo.
\end{abstract}

Methods Using hybridoma technology, we generated a specific monoclonal antibody (mAb) against recombinant human RANK receptor and characterized by SDS PAGE, ELISA, Western Blot and immunocytochemistry, then synthesized osteoclast-targeting bioconjugates of salmon calcitonin ( $\mathrm{SCT}$ ) using this antibody by generating thiol groups on $\mathrm{mAb}$ using 2-Iminothiolane and subsequently reacting them with sCT-PEG-MAL synthesised from $\mathrm{sCT}$ and NHS-PEG-MAL. To test the efficacy of the conjugate in vitro, osteoclasts were generated from precursor RAW 264.7 cells by dosing with the cytokines macrophagecolony-stimulating factor (M-CSF), and RANK Ligand (RANKL) and TRAP activity assay, Resorption Pit Assay, TRAP staining were performed. Cytotoxicity of the mAbsCT conjugate was also evaluated in RAW 264.7 cells; sCT bioactivity and CTR binding potential were evaluated by in vitro intracellular CAMP stimulation assay in human T47D breast cancer cells.

M. Newa $\cdot$ K. H. Bhandari $\cdot$ L. Tang $\cdot$ R. Kalvapalle $\cdot$ M. Suresh .

M. R. Doschak $(\triangle)$

Faculty of Pharmacy \& Pharmaceutical Sciences, University of Alberta

Edmonton, Alberta T6G 2N8, Canada

e-mail: mdoschak@ualberta.ca

M. Newa

e-mail: newa@ualberta.ca

K. H. Bhandari

e-mail: kbhanda@ualberta.ca
Results Generation of antibody against human RANK receptor was confirmed by SDS PAGE, ELISA and Western Blot. Immunocytochemistry confirmed the osteoclast targeting potential of the antibody. Successful conjugation of the antibody with sCT was confirmed by SDS PAGE and ELISA. Multinucleated osteoclast formation was confirmed by staining for tartrate-resistant acid phosphatase (TRAP). Conjugate functionality was confirmed by TRAP activity and Resorption Pit assay, showing the inhibitory effect on osteoclast differentiation. CAMP assay confirmed the retention of calcitonin bioactivity after conjugation.

Conclusions Our strategy offers the potential for a "universal" osteoclast-targeting platform - one that targets the RANK receptor on osteoclast cells by simply altering the conjugated cargo in order to affect the specific regulation of osteoclast cells.

KEY WORDS drug delivery system · monoclonal antibody. osteoclast - osteoporosis · post-traumatic osteoarthritis .

RANK receptor s salmon calcitonin

\section{INTRODUCTION}

Osteoclasts are the cells uniquely responsible for dissolving both the organic and inorganic components of bone during

\author{
L. Tang \\ e-mail: xinli@ualberta.ca \\ R. Kalvapalle \\ e-mail: rohitk@ualberta.ca \\ M. Suresh \\ e-mail: msuresh@pharmacy.ualberta.ca
}


development and are part of the bone remodeling cycle throughout life. These cells originate from hematopoietic precursors of the monocyte/macrophage lineage that are present both in the bone marrow and peripheral circulation, and their numbers and/or activity are frequently increased in a wide range of clinical disorders that are associated with excessive bone loss and which affect millions of people (1).

Osteoporosis is a major metabolic bone disease that predisposes patients to fracture in up to $40 \%$ of aging women and $15 \%$ of aging men (2). The increase in bone resorption is due both to increased osteoclastogenesis and to decreased osteoclast apoptosis (2,3). Osteoclasts may also play a role in the etiology of post-traumatic osteoarthritis (PTOA), the most common form of arthritis. At an early stage of disease pathogenesis, there is a phase of increased bone resorption and turnover in periarticular subchondral bone volume and an increased number of osteoclasts, prior to later stage sclerosis and eburnation (4). Similarly, increased osteoclast functional activity is directly responsible for the generalized bone loss that occurs in rheumatoid arthritis (5). Thus, we hypothesized that an antiresorptive strategy that selectively targets osteoclasts and/or carries an active drug to osteoclast cells directly would be highly desirable as a therapeutic to treat bone disease involving upregulated osteoclast activity.

Osteoclast cells express the RANK receptor (receptor activator of nuclear factor Kappa B) and in effect serve as ideal molecular targets. RANK is expressed on osteoclast precursors as well as mature osteoclasts. RANK is the essential signaling receptor for osteoclast differentiation during the process of osteoclastogenesis, as triggered by the osteoclast differentiation factor known as RANK-ligand (RANKL) (6,7). RANK signaling, with additional signaling through c-Fms, the receptor for macrophage-colony-stimulating factor (M-CSF), triggers the proliferation and fusion of mononuclear cells and the formation of multinucleated, mature osteoclasts $(7,8)$.

Mature osteoclasts will also express the calcitonin receptor upon its surface, which has been known for decades as a prominent negative controller of bone resorption (9). Calcitonin receptors are of the $\mathrm{G}$ proteincoupled receptor family (comprising seven transmembranespanning receptor domains) whose signaling will inhibit osteoclast activity both in vitro and in vivo (10). Calcitonin receptor activation upon osteoclasts, by its ligand calcitonin, will rapidly induce the loss of ruffled border and immobility followed by cell retraction and arrest of bone resorption. Calcitonin receptor signaling will also alter ion transporter distribution, impair enzyme activity (11) and inhibit the osteoclastogenic effects of RANKL (12).
Since antibodies have exquisite specificity of target recognition and, thus, generate highly selective outcomes following their systemic administration, our purpose was to develop a "universal" osteoclast-targeting platform as a drug-delivery strategy in order to deliver antiresorptive drugs using an anti-RANK mAb capable of localizing to the cells responsible for bone resorption. We have chosen salmon calcitonin as a model drug, as it acts on the calcitonin receptor also found on bone-resorbing osteoclasts.

\section{MATERIALS AND METHODS}

\section{Preparation of Anti-RANK Monoclonal Hybridoma Cell Lines}

Monoclonal antibody (mAb) to the RANK receptor was generated using Hybridoma technology, as previously performed in our laboratory $(13,14)$. Briefly, 6-8-week-old female $\mathrm{BALB} / \mathrm{c}$ mice were immunized intraperitoneally three times with $25 \mu \mathrm{g}$ of recombinant human sRANK receptor (Peprotech, USA) on days 0 and 14 using complete and incomplete Freund's adjuvant, respectively, and once with $10 \mu \mathrm{g}$ of antigen on day 28 using phosphate-buffered saline (PBS, pH 7.3). The immune response to the antigen was assessed by measuring the titer of polyclonal antibody in mouse serum using an indirect enzyme-linked immunosorbent assay (ELISA). Mice with the highest antibody titer were euthanized and splenectomized 3 days after the final injection of antigen. Spleen cells were fused with SP2/0 myeloma cells at a ratio of $4: 1$ using $50 \%(\mathrm{w} / \mathrm{v})$ polyethylene glycol (PEG) (Sigma, USA) according to the technique described previously by Köhler \& Milstein (15). The hybridoma cells were suspended in culture medium (RPMI 1640) supplemented with penicillin, streptomycin, L-glutamine (PSG), hypoxanthine aminopterin thymidine (HAT), and 20\% fetal bovine serum (FBS) (Sigma, USA). Cells were seeded in 96-well tissue culture plates and incubated in a humidified $37^{\circ} \mathrm{C}, 5 \%$ carbon dioxide incubator for 2 weeks. Clones were maintained in HAT medium for a further 2 weeks.

Hybridoma cell lines were screened by an indirect ELISA. The cell lines producing specific antibodies were recloned successively 3-7 times by limiting dilution to ensure monoclonality and stability of the cell line. Hybridoma cell lines were then propagated in large $175 \mathrm{~cm}^{2}$ tissue culture flasks, and the conditioned supernatant collected. Purification of IgG mAbs was achieved by affinity chromatography using Protein G agarose (Sigma, USA). The IgG immunoglobulin subclass was determined using the mouse hybridoma isotyping 
reagents according to instructions from the manufacturer (Sigma, USA).

\section{Indirect Enzyme-Linked Immunosorbent Assay (ELISA)}

All ELISAs were performed in flat-bottomed 96-well plates (Nunc-Immuno MaxisorbTM plates, Nunc). Antibody secretion by hybridoma cells was detected by indirect ELISA. Briefly, $100 \mu \mathrm{l}$ of antigen (human sRANK receptor) was used for coating at a concentration of $1 \mu \mathrm{g} / 100 \mu \mathrm{l}$, overnight at $4{ }^{\circ} \mathrm{C}$. The wells were washed three times with PBS (pH 7.3) and, to avoid nonspecific binding, incubated with $3 \%$ BSA for $1 \mathrm{~h}$ at room temperature. After washing, the wells were incubated with $100 \mu \mathrm{l}$ supernatant from each hybridoma clone for $1 \mathrm{~h}$ at room temperature. After washing, bound antibodies were detected using secondary goat anti-mouse IgG conjugated with horseradish peroxidase (GAM-HRPO) at a 1:5000 dilution for $1 \mathrm{~h}$ at room temperature. After final washing, $100 \mu \mathrm{l}$ of 3,3',5,5'tetramethylbenzidine (TMB substrate) was added to each well and incubated for $15 \mathrm{~min}$ at room temperature. The optical density (OD) was measured at $650 \mathrm{~nm}$ using an ELISA Vmax kinetic microplate reader (Molecular Devices Corp., Galifornia, USA). The clones showing ELISA values five times higher than the negative control were considered positive. Sera of unimmunized mice and irrelevant antibody were used as negative controls. RPMI media and positive sera from hyperimmunized mice were used as blank and positive control, respectively.

\section{Characterization of Anti-RANK Monoclonal Antibodies}

Sodium dodecyl sulfate polyacrylamide gel electrophoresis (SDS-PAGE) was performed on purified antibody under both reducing and non-reducing conditions. Antibody was mixed with loading buffer and run in 10\% polyacrylamide gel, with the thiol reagent 2-Mercaptoethanol added to the loading buffer for reducing conditions (to cleave the disulfide bonds between the polypeptides). Gels were stained with Coomassie blue for protein band detection of individual heavy and light chains of the antibody.

ELISA was performed as above, using $100 \mu \mathrm{l}$ RANK receptor for coating at a concentration of $1 \mu \mathrm{g} / 100 \mu \mathrm{l}$, overnight at $4^{\circ} \mathrm{C}$. To avoid nonspecific binding, the wells were incubated with $3 \%$ BSA for $1 \mathrm{~h}$ at room temperature. After washing, the wells were incubated with different concentrations of antibody for $1 \mathrm{~h}$. The wells were washed with PBS, and the bound antibodies were detected using secondary antibody (goat anti-mouse IgG conjugated with horseradish peroxidase) for $1 \mathrm{~h}$. After final washing, $100 \mu \mathrm{l}$ of TMB substrate was added to each well and incubated for $15 \mathrm{~min}$. The optical density (OD) was measured at $650 \mathrm{~nm}$.

For Western blot analysis, RANK receptor $(19.3 \mathrm{kDa})$ was electrophoresed on SDS-PAGE using 10\% acrylamide gel and transferred onto nitrocellulose membrane using mini trans-blot apparatus. The membrane was blocked with 5\% skim milk in PBS-T for $1 \mathrm{~h}$, washed with PBS-T, cut into strips, and incubated with purified $\mathrm{mAb}$ solution for $1 \mathrm{~h}$. After washing, strips were reacted with GAM-HRPO for $1 \mathrm{~h}$, and the binding of mAbs to RANK receptor was detected using enhanced chemiluminescence. For the negative control, the primary antibody was omitted, and the antigen strip incubated with PBS.

\section{Immunocytochemistry}

To confirm the RANK receptor targeting potential of generated $\mathrm{mAb}$ in vitro, osteoclast-like cells were generated in Lab Tek II chamber slide system (Nunc) from RAW 264.7 cells, as described below for generation of osteoclasts. The MG-63 osteoblast-like cell line known to lack RANK receptor (16) was used as negative control. Likewise, osteoclast cell culture omitting primary $\mathrm{mAb}$ was also used as a method negative control. Cell cultures were washed with PBS, fixed in 4\% paraformaldehyde in PBS ( $\mathrm{pH} 7.4$ ) for $5 \mathrm{~min}$ and rinsed thoroughly. After blocking with $3 \%$ BSA in PBS for $1 \mathrm{~h}$, the cells were incubated with our antiRANK $\mathrm{mAb}(4 \mu \mathrm{g} / \mathrm{ml})$ overnight at $4^{\circ} \mathrm{C}$. Antibody detection was carried out using rabbit anti-mouse IgGFITC (1:100; Sigma, USA) for $60 \mathrm{~min}$ at $4^{\circ} \mathrm{C}$. To visualize cell nuclei, slides were counterstained with $1.5 \mu \mathrm{g} / \mathrm{ml} 4-6$ diamidino-2 phenylindole (DAPI, Sigma) diluted in PBS. Culture slides were separated from their chambers, mounted and photographed using confocal microscopy (Zeiss LSM 710 with ZEN software and the microscope the Observer.Z1).

\section{Synthesis of Anti-RANK mAb-Calcitonin Conjugate}

\section{Thiolation of Antibodies Using Traut's Reagent}

In order to generate anti-RANK mAb-salmon calcitonin conjugate $(\mathrm{mAb}-\mathrm{sCT})$, free thiol groups were generated on our anti-RANK $\mathrm{mAb}$ by reacting it in a 10 molar excess of Traut's reagent at room temperature for $1.5 \mathrm{~h}$ (Fig. 1a). Traut's reagent (2-Iminothiolane or 2-IT, Sigma USA), a cyclic thioimidate compound, reacts spontaneously and efficiently with primary amines at $\mathrm{pH}$ 7-9 resulting in sulfhydryl addition $(17,18)$. No free sulfhydryl moieties were 
found to be present in our IgG mAb, as determined by Ellman's assay (19).

\section{Synthesis of Thiol-Reactive Calcitonin Analogue}

To generate thiol-reactive calcitonin analogue, synthetic salmon calcitonin (sCT, Calbiochem, USA) in DMSO $(13.72 \mathrm{mg} / \mathrm{ml}$ ) was mixed with NHS-PEG-MAL (Creative Biochem, USA) in DMSO $(51 \mathrm{mg} / \mathrm{ml})$ (Sigma,USA) in 1:3 molar ratio. DMSO was chosen as the reaction medium because of the instability of NHS and sCT in aqueous solutions. sCT is further highly soluble and highly stable in DMSO (20). The reaction between the primary amines in sCT and NHS group of NHS-PEG-MAL was allowed to proceed at room temperature with constant stirring for 45 min. sCT has three primary amines at residues Lys 11 , Lys 18 and the N-terminus, which can react with the NHS functional group of NHS-PEG-MAL to generate three intermediate conjugates: mono-, di- and tri-substituted thiol reactive $\mathrm{sCT}$ analogues (Fig. 1b).

(a)<smiles>[R]NC(CCCC)CCCS</smiles>

Traut's Reagent Primary Amine Modification Producing a Molecule Terminal Sulfhydryl Group

Structure of Traut's Reagent and reaction scheme with molecules containing primary amines.

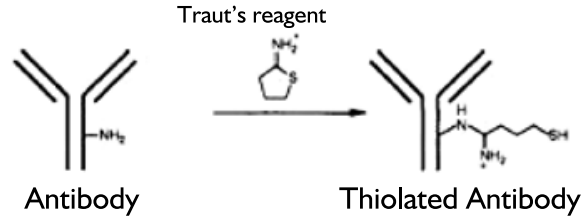

(b)

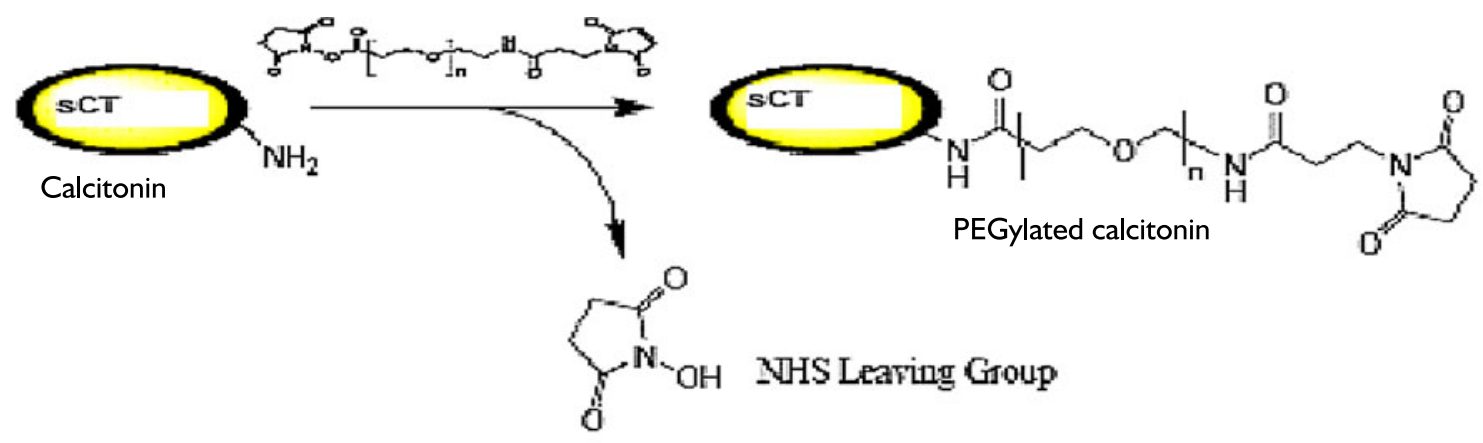

(c)

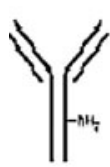

Anti-

RANK

MAB

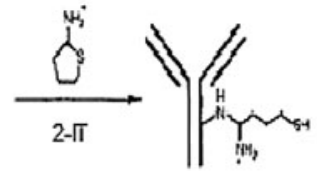

Thiolated

mAb

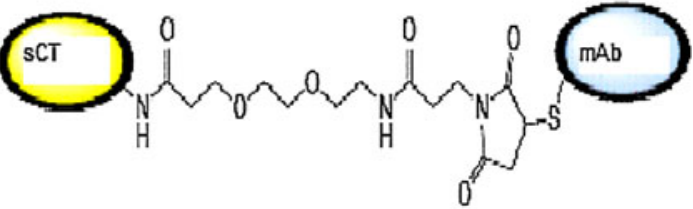

sCT-PEG-Antibody

Fig. I (a) Thiolation of antibodies using Traut's reagent. (b) Synthesis of thiol reactive sCT analogue by reacting sCT with NHS-PEG-MAL. c Coupling free thiol containing $\mathrm{mAb}$ with functionalized thiol reactive $\mathrm{sCT}$ analogue. 


\section{Coupling Free Thiol-Containing mAb with Functionalized Thiol-Reactive sCT Analogue}

Functionalized thiol-reactive sCT analogue was added intermittently with constant stirring to thiol-mAb solution at 10:1 molar ratio, and the reaction between the thiolreactive MAL groups in functionalized sCT and $\mathrm{SH}$ group of thiol-mAb was allowed to proceed at room temperature in the dark with constant stirring for $2 \mathrm{~h}$. Unreacted sCTPEG was removed by dialysis at $4^{\circ} \mathrm{C}$ (Fig. 1c).

\section{Characterization of Anti-RANK mAb-sCT Conjugate}

Anti-RANK mAb-sCT conjugate was examined under both reducing and non-reducing conditions of SDSPAGE analysis, as described above. ELISA was performed, using $100 \mu \mathrm{l}$ RANK receptor for coating at a concentration of $1 \mu \mathrm{g} / 100 \mu \mathrm{l}$, overnight at $4^{\circ} \mathrm{C}$. To avoid nonspecific binding, the wells were incubated with $3 \%$ BSA for $1 \mathrm{~h}$ at room temperature. The wells were incubated in triplicate with either $\mathrm{mAb}$ alone, functionalized sCT-PEG, or mAb-sCT conjugate for $1 \mathrm{~h}$. The wells were washed with PBST followed by incubation with rabbit anti-salmon calcitonin antibody (US Biologicals) for $1 \mathrm{~h}$. The wells were washed with PBST three times and incubated with HRPO conjugated goat antirabbit IgG (R\&D Systems) for $1 \mathrm{~h}$. After final washing with PBST, TMB substrate was added to each well, and the OD measured at $650 \mathrm{~nm}$.

\section{In Vitro Testing of mAb-sCT Conjugate Efficacy}

\section{Generation of Osteoclasts}

Osteoclast-like cells were generated in culture from RAW 264.7 cells (transformed murine monocytic cell line), purchased from the American type culture collection (ATCG, VA, USA) (21). RAW 264.7 cells were cultured to confluence in a $75 \mathrm{~cm}^{2}$ flask in GIBCO High Glucose 1X Dulbecco's Modified Eagle Medium (DMEM, Invitrogen, CA, USA) containing $4.5 \mathrm{~g} / \mathrm{L}$ D-Glucose, Lglutamine, and $110 \mathrm{mg} / \mathrm{L}$ sodium pyruvate, with the addition of $10 \%$ heat-inactivated fetal bovine serum (FBS) and $1 \%$ penicillin-streptomycin added $(10,000 \mathrm{U} / \mathrm{mL}$; Invitrogen, USA). Confluent cells were harvested by scraping, centrifuged at 1,500 RPM, and resuspended in $25 \mathrm{~mL}$ of culture medium. The number of cells in the suspension was measured using a Bright-Line Hemocytometer (Hausser Scientific, PA, USA) and seeded at a concentration of $2 \times 10^{3}$ cells/well in 96-well culture plates (Corning) and placed in the $\mathrm{CO}_{2}$ incubator overnight to allow the cells to attach to the surface. After $24 \mathrm{~h}$, the culture medium was replaced with media containing
$25 \mathrm{ng} / \mathrm{ml}$ macrophage-colony-stimulating factor (M-CSF) and $50 \mathrm{ng} / \mathrm{mL}$ RANKL (PeproTech, NJ, USA). Osteoclasts were successfully generated by dosing with M-CSF and RANKL every $48 \mathrm{~h}$ over the course of 7 days. To confirm the generation of multinucleated osteoclast-like cells, the cultured cells were stained for the enzyme tartrate-resistant acid phosphatase (TRAP) using the Leukocyte Acid Phosphatase TRAP Kit from SigmaAldrich (St.Louis, MO, USA), according to the manufacturer's instructions. TRAP-positive multinucleated osteoclasts were visualized by light microscopy and photographed.

\section{Spectrophotometric Assay of In Vitro TRAP Activity}

To quantify the total TRAP activity from in vitro osteoclast cultures, RAW264.7 cells were seeded at a concentration of $2 \times 10^{3}$ cells/well in 96-well culture plates and incubated for $24 \mathrm{~h}$. Peptide factors $50 \mathrm{ng} / \mathrm{ml}$ RANKL and MCSF $25 \mathrm{ng} / \mathrm{ml}$ were added to the culture, with or without the addition of $100 \mathrm{nM}$ of $\mathrm{mAb}, 100 \mathrm{nM}$ of mAb-sCT, or $100 \mathrm{nM}$ of sCT. The medium and factors were replaced every $48 \mathrm{~h}$. Osteoclastogenesis was assessed by the spectrophotometric measurement of TRAP activity on day 7. Briefly, the medium was aspirated, and the cell monolayer washed twice with PBS. The cells were then lysed with $100 \mu \mathrm{l}$ of $0.2 \%$ Triton X-100 in water $(\mathrm{v} / \mathrm{v})$ for $10 \mathrm{~min}$. TRAP activity in the cell lysate was determined using an Acid Phosphatase Assay Kit (Cayman chemical, USA). The assay utilizes para-nitrophenyl phosphate (pNPP) as a chromogenic substrate for the TRAP enzyme. In the first step, acid phosphatase dephosphorylates pNPP. L-tartrate, an inhibitor of nontartrate resistant acid phosphatase, provided in the kit was used to measure TRAP enzyme activity. In the second step, the phenolic OH- group was deprotonated under alkaline conditions, resulting in p-nitrophenolate that yields an intense yellow color, which was measured at $405 \mathrm{~nm}$ using a microplate reader.

\section{In Vitro Mineral Resorption Assay}

To quantify the mineral resorptive activity of osteoclast-like cells in culture, RAW264.7 cells were suspended in cell culture medium and seeded in a 16-well BD Biosciences Osteologic ${ }^{\circ}$ Slide (BD Biosciences, MA, USA) at the density of $2 \times 10^{3}$ cells/well and incubated for $24 \mathrm{~h}$. Osteoclast-like cells were successfully generated by dosing with $50 \mathrm{ng} / \mathrm{ml}$ RANKL and $25 \mathrm{ng} / \mathrm{ml}$ M-CSF every $48 \mathrm{~h}$ over the course of 7 days. The wells were incubated with $100 \mathrm{nM}$ of either mAb alone, sCT alone, or mAb-sCT conjugate. Half the volume of the medium and factors were replaced every $48 \mathrm{~h}$. After 7 days of culture, the 
medium was aspirated, the slide placed in a petri-dish and washed with double-distilled water. To remove the adherent cells, the slide was then soaked in bleach for $10 \mathrm{~min}$ and washed with double-distilled water. Slides were air-dried, viewed under an inverted light microscope, and low power images of the calcium phosphate mineral remaining on the slide acquired. To quantify the resorptive effect of osteoclasts, the images were analyzed using the javabased image-processing program ImageJ (NIH, USA).

\section{Cytotoxicity Test for mAb-sCT Conjugate}

To ensure that the mAb-sCT conjugate was not cytotoxic to cells other than osteoclasts, we employed the MTT assay, in which mitochondrial dehydrogenases of viable cells cleave the tetrazolium ring of MTT, yielding purple formazan crystals. An increase or decrease in cell number results in a concomitant change in the amount of formazan formed, indicating the degree of cytotoxicity caused by the test material.

RAW 264.7 cells were seeded on 96-well plates at an initial density of $2 \times 10^{3}$ cells/well and incubated for $72 \mathrm{~h}$ at $37^{\circ} \mathrm{C}, 5 \% \mathrm{CO}_{2}$ until the cells were $80 \%$ confluent. The culture medium was replaced by $200 \mu \mathrm{l}$ basic DMEM media without FBS and incubated for $30 \mathrm{~min}$. Then the culture medium was replaced by $100 \mu \mathrm{l}$ basic DMEM media containing 50, 100 and $200 \mathrm{nM}$ of mAb, mAb-sCT and $\mathrm{sCT}$, respectively (in quadruplicate). The cells were incubated for $4 \mathrm{~h}$ at $37^{\circ} \mathrm{C}$. The medium was then replaced with $100 \mu \mathrm{l}$ basic medium containing (3-[4,5-dimethylthiazol2-yl]-2,5-diphenyl tetrazolium bromide) (MTT) at a concentration of $100 \mu \mathrm{g} /$ well and incubated for a further $4 \mathrm{~h}$ at $37^{\circ} \mathrm{C}$. After removing the supernatant and washing twice with phosphate-buffered saline, the newly formed purple formazan crystals were dissolved in $200 \mu$ l solubilization solution using an in vitro toxicology assay kit (TOX-1, Sigma Aldrich, St. Louis, USA), and the absorbance was measured at $570 \mathrm{~nm}$ using a microplate reader.

\section{In Vitro Bioactivity of $\mathrm{mAb}-\mathrm{sCT}$ and Calcitonin Receptor Binding Affinity}

To confirm that calcitonin bioactivity was not lost after conjugation to $\mathrm{mAb}$ and to confirm the ability for $\mathrm{mAb}$ sCT conjugate to trigger the calcitonin receptor, T47D human breast cancer cells (known to contain calcitonin receptors) were used, as previously described (22). Briefly, T47D cells (ATCG, VA, USA) were cultured in triplicate in RPMI-1,640 culture medium containing $1 \%$ penicillinstreptomycin, $10 \%$ fetal bovine serum, and insulin (0.2 IU/ $\mathrm{mL}$ ). Cells were seeded on 48-well plates at an initial density of $5 \times 10^{4}$ cells/well and incubated in 95\% air and 5\% $\mathrm{CO}_{2}$ at $37^{\circ} \mathrm{C}$ for 2 days. Cells were then washed with HBSS and preincubated in RPMI-1640 culture medium devoid of FBS, insulin and antibiotics. Cells were dosed with the phosphodiesterase inhibitor, 3-isobutyl-1-methyl-xanthine (IBMX, $1 \mathrm{mM}$ ) and incubated at $37^{\circ} \mathrm{C}$ for $30 \mathrm{~min}$. One hundred $\mathrm{nM}$ of $\mathrm{mAb}, \mathrm{mAb}-\mathrm{sCT}$ and $\mathrm{sCT}$ were then added to the wells except the control where T47D cells were treated with IBMX only. The plate was incubated for $20 \mathrm{~min}$ at $37^{\circ} \mathrm{C}$.

After removing the supernatant, cells were washed three times in cold phosphate-buffered saline and resuspended in $500 \mu \mathrm{l}$ of cell lysis buffer. Cells were frozen at $-20^{\circ} \mathrm{C}$ and thawed with gentle mixing. The freeze/thaw cycle was repeated three times, and the mixture was centrifuged at $600 \mathrm{~g}$ for $10 \mathrm{~min}$ at $2-8^{\circ} \mathrm{C}$ to remove cellular debris. The supernatant was collected and stored at $-20^{\circ} \mathrm{C}$. cAMP concentrations were then measured using the cyclic adenosine monophosphate (cAMP) Enzyme ImmunoAssay (EIA) kit (KGE002B, R \& D systems, USA).

\section{STATISTICAL ANALYSIS}

Statistical analysis was conducted using Graph Pad Prism 5 (GraphPad Software Inc., CA). Results are presented as the mean \pm standard deviation of three samples. Unpaired t-tests were used to assign significance between groups, with a P-value of less than 0.05 as the threshold for significance. Two-way ANOVA was used for the MTT assay.

\section{RESULTS}

\section{SDS-PAGE Analysis}

A band at $150 \mathrm{kDa}$ was seen for unconjugated anti-RANK $\mathrm{IgG}$, with separate bands for heavy chain at $50 \mathrm{kDa}$ and light chain at $25 \mathrm{kDa}$ observed under reducing condition. For mAb-sCT conjugate, a band above $150 \mathrm{kDa}$ was observed, confirming the conjugation to have taken place. Under reducing condition of SDS-PAGE for conjugate, some bands above $50 \mathrm{kDa}$ and $25 \mathrm{kDa}$ were observed, suggestive of conjugation of PEGylated calcitonin to the heavy and light chains of the antibody. (Fig. 2)

\section{ELISA}

The optical density was seen to linearly increase with the concentration of mAb (Fig. 3a). Spectrophotometric absorbance value for $0.25,0.5,1.0,2.0$ and $4.0 \mu \mathrm{g}$ of the antiRANK antibody was $0.025,0.070,0.131,0.27$ and 0.518 , respectively. 
ELISA carried out for the conjugate showed the binding of the conjugated antibody to the coated RANK receptor. The $\mathrm{SCT}$ attached to the conjugate was detected using an anti-calcitonin antibody, thus confirming the intactness of mAb-sCT conjugates. Optical density for $\mathrm{mAb}$ alone was negligible, as it did not have calcitonin to be detected by anti-calcitonin antibody. Similarly, OD for sCT-PEG was also significantly low, as it did not have the anti-RANK mAb targeting moiety necessary to bind to the RANK coated plate (Fig. 3b). ELISA-derived spectrophotometric absorbance values for $\mathrm{mAb}$, sCT-PEG and mAb-sCT conjugate were 0.002, 0.025 and 0.153 , respectively.

\section{Western Blot Analysis}

Recombinant human sRANK receptor is a $19.3 \mathrm{kDa}$ polypeptide. It was electrophoresed on a $10 \%$ polyacrylamide gel and transferred to nitrocellulose membrane. On incubation with generated $\mathrm{mAb}$, a band was detected between 15 and $20 \mathrm{kDa}$, after the chemiluminescent detection of anti-RANK IgG (Fig. 4).

\section{Osteoclast Generation and Confirmation by TRAP Staining}

Osteoclasts were generated by dosing the RAW264.7 cells with RANKL and MCSF. The staining for TRAP is a technique commonly used to visualize osteoclasts. The principle behind staining of TRAP involves the use of napthol AS phosphates in conjunction with fast garnet GBC salts for the detection of acid phosphatase. This diazonium fast garnet GBC salt was selected because it couples rapidly at acid $\mathrm{pH}$, forming insoluble dye deposits. Napthol AS-BI, released by enzymatic hydrolysis, couples immediately with fast garnet GBC salt, resulting in the (a)

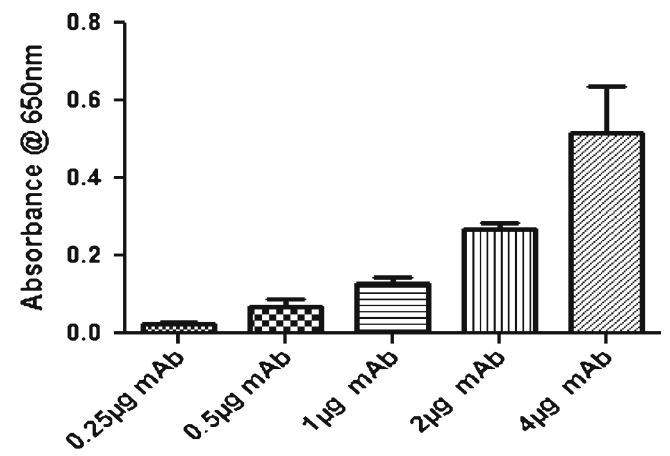

(b)

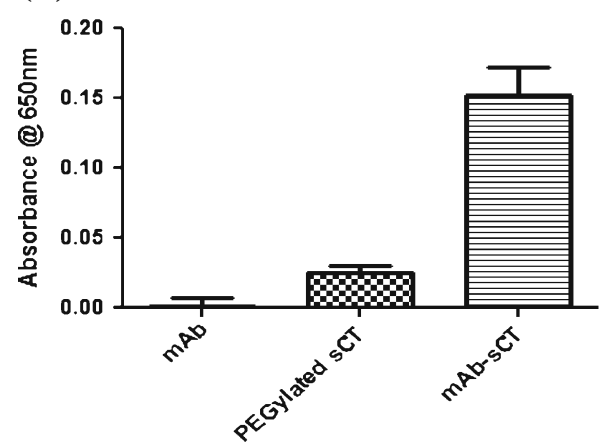

Fig. 3 (a) ELISA conducted on RANK receptor-coated plate. The optical density was seen to be proportional with the concentration of anti-RANK antibody. (b) ELISA of the mAb-sCT conjugate where anti-Calcitonin secondary antibody reagent was used to detect immobilized Calcitonin residues that were part of the primary anti-RANK conjugates and that were binding to the RANK-coated wells. Optical density for mAb alone was negligible as it did not have calcitonin to be detected by anti-calcitonin antibody. Similarly, OD for sCT-PEG was also significantly low as it did not have anti-RANK antibody to bind to RANK-coated plate.
Fig. 2 SDS-PAGE analysis of mAb-sCT conjugate and unconjugated antibody. A protein band above $150 \mathrm{kDa}$ was seen for $\mathrm{mAb}-\mathrm{s} C \mathrm{C}$ conjugate in non-reduced SDS-PAGE analysis. Some bands above $50 \mathrm{kDa}$ as well as $25 \mathrm{kDa}$ were observed for the conjugate in reduced SDS PAGE. Lane I: unconjugated antibody (non-reducing condition), Lane 2: mAb-sCT conjugate (nonreducing), Lane 3, 6:

standard marker, Lane 4: mAb-sCT conjugate (reducing), Lane 5: unconjugated antibody (reducing).
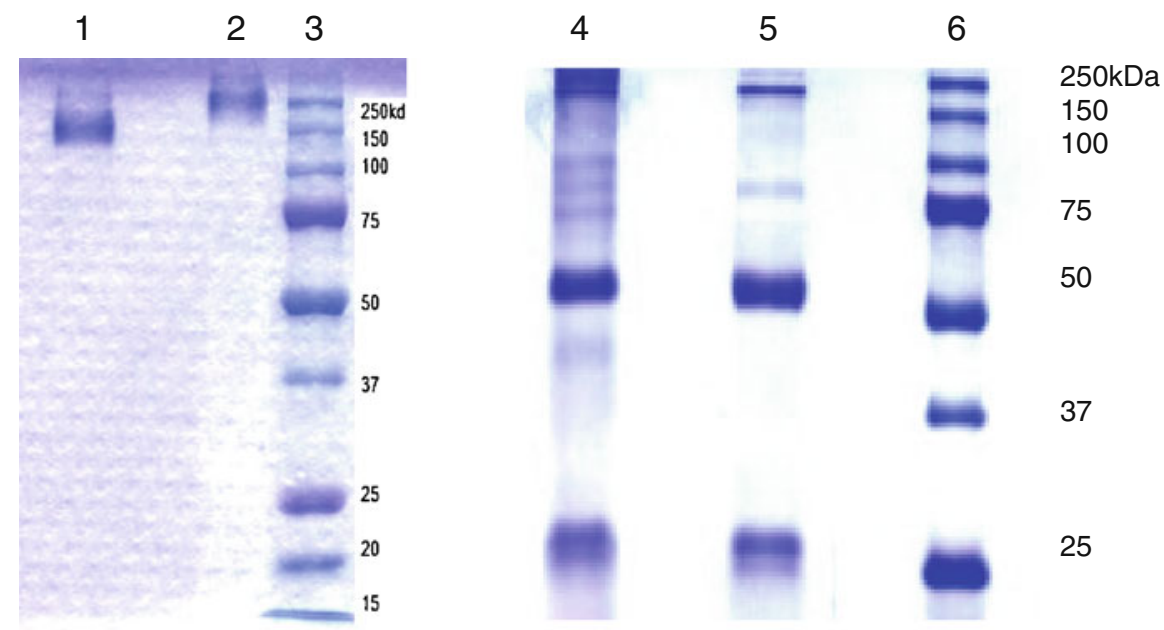


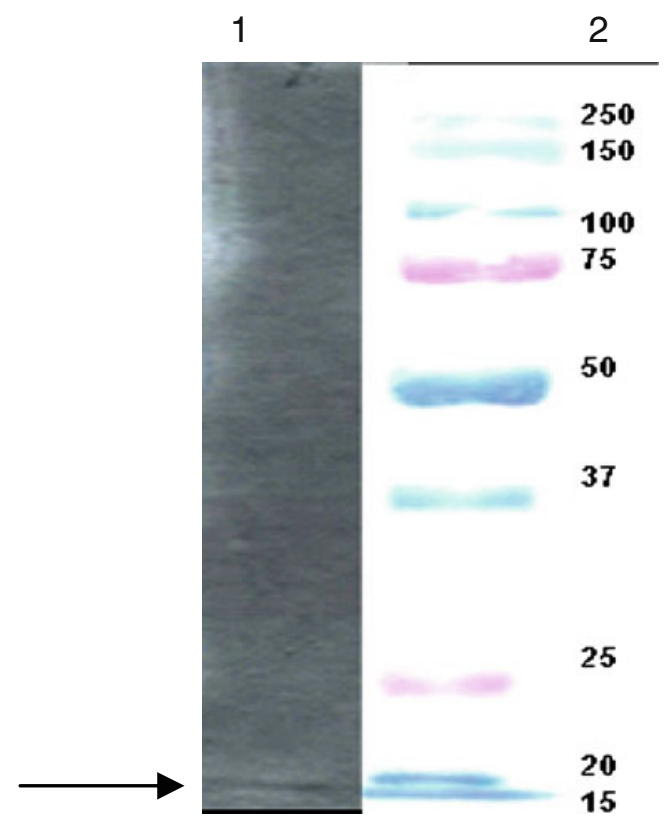

Fig. 4 Western Blot Analysis: Lane I: a band seen between $20 \mathrm{kDa}$ and $15 \mathrm{kDa}$ as a result of chemiluminescent detection of anti-RANK IgG. Lane 2: standard marker. RANK receptor $(19.3 \mathrm{kDa})$ was electrophoresed on $10 \%$ SDS-PAGE. Protein was transferred to nitrocellulose membrane which was blocked with $5 \%$ skim milk, followed by the incubation with anti-RANK mAb. Reaction was detected by using GAM-HRPO, and the membrane was developed using ECL reagent and exposure to $X$-ray film.

formation of insoluble maroon deposits at sites of activity. Tartaric acid was used in order to demonstrate the presence of tartrate-resistant acid phosphatase. Hence, cells containing tartrate-sensitive acid phosphatase are devoid of activity, and only the cells containing tartrate acid-resistant phosphatase show maroon dye deposits at the sites of activity. Staining confirmed that the presence of TRAP-positive multinucleated osteoclastlike cells was limited to those wells dosed with exogenous M-CSF and RANKL, under the cell culture conditions described (Fig. 5).

\section{Immunocytochemistry}

Confocal microscopy confirmed that the anti-RANK receptor mAb staining (green color) was limited to surface receptors present on osteoclastic precursors as well as mature osteoclast-like cells (Fig. 6a). Counterstaining with DAPI (blue color) confirmed the multinucleated phenotype of osteoclast-like giant cells. Nucleoli were also witnessed in the images. There was no demonstrable fluorescent fluorescein isothiocyanate (FITC) staining in negative control slides (Fig. 6b and c). A commercially available anti-RANK mAb was used as a positive method control to confirm that RAW 264.7 osteoclastic precursors as well as mature osteoclast-like cells indeed stained for surface RANK receptors using immunocytochemistry.

\section{TRAP Activity Assay}

RAW 264.7 cell cultures treated with exogenous RANKL and MCSF showed a 9.9-fold increase of TRAP activity compared to controls without cytokine treatment. We found that the release of this osteoclast-associated enzyme was potently inhibited by our mAb-sCT conjugate (and by our unconjugated $\mathrm{mAb}$ alone), as compared to sCT alone (Fig. 7). We measured a 5.47-fold decrease in TRAP activity after treatment with $\mathrm{mAb}-\mathrm{sCT}$ conjugate. Also, the conjugate showed a 2.82-fold greater inhibition of TRAP compared to sCT treatment alone.

\section{In Vitro Mineral Resorption Assay}

The osteoclast-like cells generated on Osteologic ${ }^{\circledR}$ calcium phosphate-coated culture wells were shown to be capable of resorbing the immediate calcium phosphate layer surrounding them. That result was in direct contrast to precursor RAW 264.7 cells (cultured in the absence of M-CSF and RANKL) that were shown not to affect the calcium phosphate coating in any manner. Osteoclast-like cell cultures treated with the $\mathrm{mAb}-\mathrm{sCT}$ conjugate, or $\mathrm{mAb}$ alone, demonstrated a significant inhibitory effect on the resorptive ability of the osteoclast-like cells, as measured by the conservation of calcium phosphate layer on the Osteologic ${ }^{\circledR}$ slide. The integrated density value (IDV) of the remaining calcium phosphate layer for the culture treated with the mAb-sCT conjugate was significantly greater than that treated with sGT alone (Fig. 8).

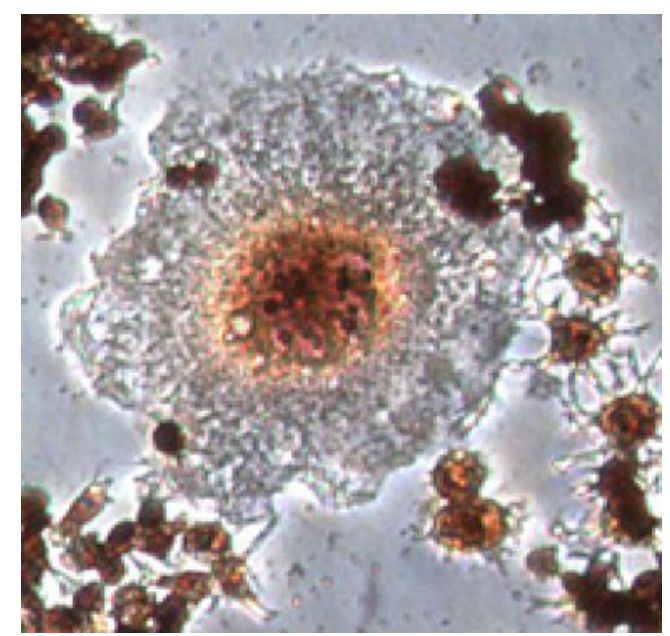

Fig. 5 Multinucleated TRAP-positive osteoclast was observed by TRAP staining. RAW 264.7cells, a murine macrophage-like osteoclast precursor line, were stimulated with $50 \mathrm{ng} / \mathrm{ml}$ RANKL and $25 \mathrm{ng} / \mathrm{ml}$ MCSF for osteoclast generation. 

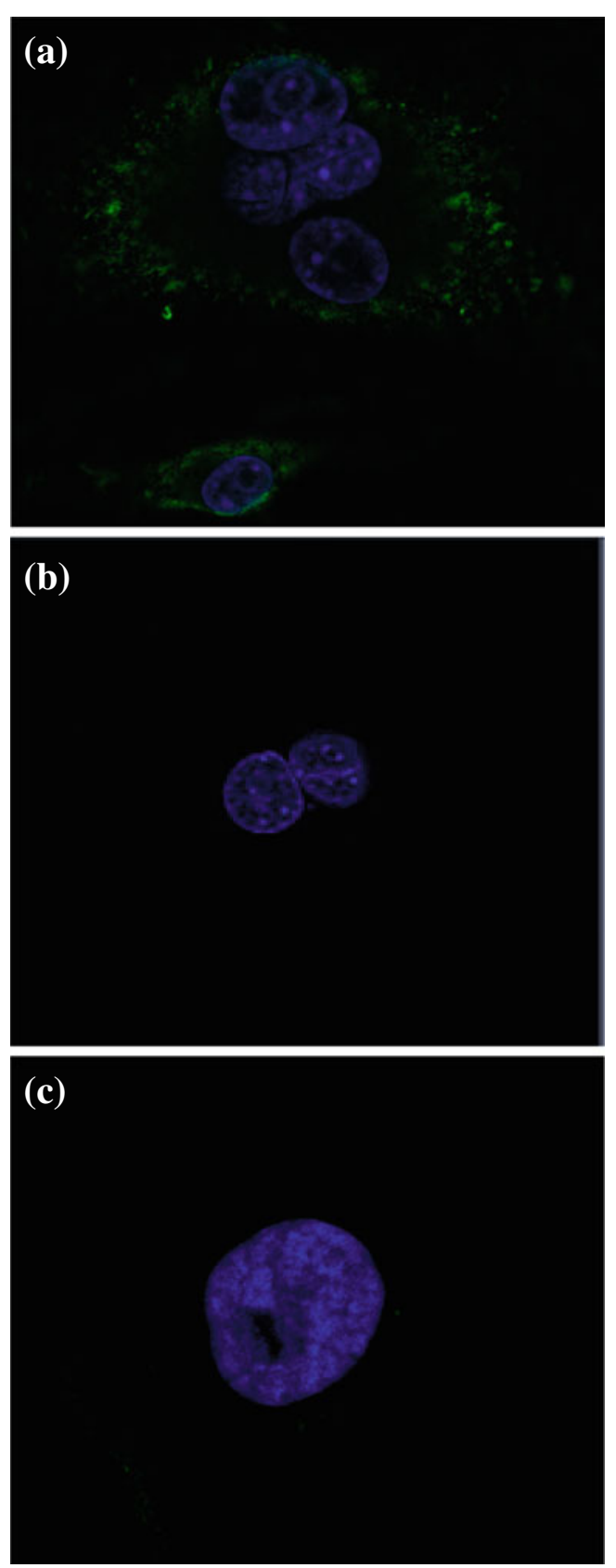

Fig. 6 (a) Confocal images of immunocytochemistry performed with anti-RANK mAb which detected RANK receptors, the surface receptors present on the osteoclasts as well as osteoclast precursors. Counterstaining with DAPI showed the multinucleation in osteoclasts. (b) Confocal image of immunocytochemistry for osteoclast cell culture omitting primary antibody, as a negative control. (c) Confocal image of immunocytochemistry for osteoblast-like MG-63 cell line as a negative control. The MG-63 cell line does not express RANK receptors.

\section{Cytotoxicity of mAb-sCT Conjugate}

To test for potential cytotoxic effects of our $\mathrm{mAb}$ and/or mAb-sCT conjugate, we tested RAW 264.7 cell viability using the MTT assay. Anti-RANK monoclonal antibody, mAbsCT conjugate and sCT showed no demonstrable cytotoxicity at the concentrations tested (up to $200 \mathrm{nM}$ ), as measured by the absorbance of formazan solution formed after $4 \mathrm{~h}$ incubation (Fig. 9). Thus, the viability of these cells was not perturbed by our $\mathrm{mAb}$ and/or conjugate treatments.

\section{In Vitro Bioactivity of mAb-sCT and Calcitonin Receptor Binding Affinity}

Using an in vitro competitive binding assay, cAMP activity was determined in TD47 breast cancer cells known to contain the calcitonin receptor. The assay confirmed that sCT and the mAb-sCT conjugate were capable of stimulating cAMP production after binding the calcitonin receptor. The significantly lower absorbance values measured (due to the competitive nature of the assay) indicated the ability for sCT and/or mAb-sCT to generate intracellular cAMP in the presence of a phosphodiesterase inhibitor. Conversely, the mAb-treated and control wells exhibited negligible intracellular cAMP generation, and, accordingly, high absorbance values in the competitive binding assay were observed (Fig. 10).

\section{DISCUSSION}

Osteoclasts are the only cells capable of resorbing mineralized bone. However, current antiresorptive therapies do not specifically target the osteoclast cell and elicit systemic side effects in cells other than osteoclasts. For example, the development of duodenal ulcers, gastric erosions and potentially even osteonecrosis of the jaw or catastrophic low energy fracture of the femoral shaft have all been

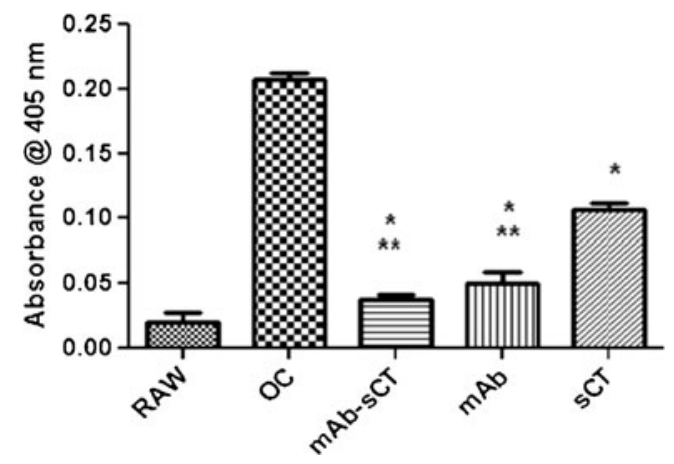

Fig. 7 Tartrate-resistant acid phosphatase (TRAP) activity assay. mAb-sCT conjugate showed an inhibitory effect on TRAP activity, an important marker of osteoclasts. RAW: cells cultured in media with $0 \mathrm{ng} / \mathrm{ml}$ RANKL $+0 \mathrm{ng} / \mathrm{ml}$ MCSF; OC: cells cultured in media with $50 \mathrm{ng} / \mathrm{ml}$ RANKL + $25 \mathrm{ng} / \mathrm{ml}$ MCSF; mAb-sCT: cells cultured in media with $50 \mathrm{ng} / \mathrm{ml}$ RANKL + $25 \mathrm{ng} / \mathrm{ml}$ MCSF + $100 \mathrm{nM}$ conjugate; mAb: cells cultured in media with $50 \mathrm{ng} / \mathrm{ml}$ RANKL $+25 \mathrm{ng} / \mathrm{ml}$ MCSF + $100 \mathrm{nM}$ $\mathrm{mAb}$; sCT: cells cultured in media with $50 \mathrm{ng} / \mathrm{ml}$ RANKL $+25 \mathrm{ng} / \mathrm{ml}$ MCSF +100 nM sCT. * $P<0.05$ versus OC; ** $P<0.05$ versus $\mathrm{sCT}$. No statistically significant difference was observed between naked antibody and the conjugate. 


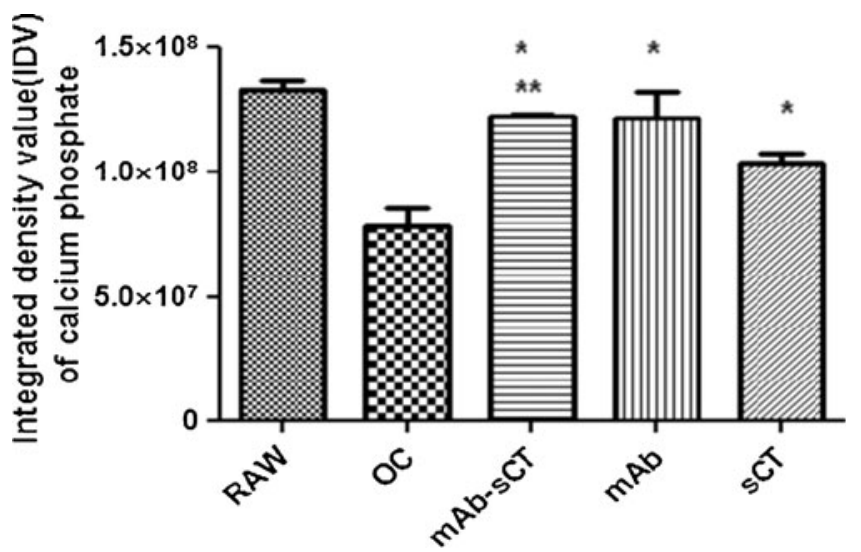

Fig. 8 Demonstration of resorption pit formation on osteologic substrate plate. After 7 days of culture, adherent cells were removed, and integrated density value of the remaining osteologic layer was calculated using alphaimager densitometer. RAW: cells cultured in media with $0 \mathrm{ng} / \mathrm{ml}$ RANKL $+0 \mathrm{ng} / \mathrm{ml}$ MCSF; OC: cells cultured in media with $50 \mathrm{ng} / \mathrm{ml}$ $\mathrm{RANKL}+25 \mathrm{ng} / \mathrm{ml} \mathrm{MCSF}$; mAb-sCT: cells cultured in media with $50 \mathrm{ng} / \mathrm{ml}$ RANKL + $25 \mathrm{ng} / \mathrm{ml}$ MCSF + $100 \mathrm{nM}$ conjugate; mAb: cells cultured in media with $50 \mathrm{ng} / \mathrm{ml}$ RANKL $+25 \mathrm{ng} / \mathrm{ml} \mathrm{MCSF}+100 \mathrm{nM}$ $\mathrm{mAb}$; sCT: cells cultured in media with $50 \mathrm{ng} / \mathrm{ml}$ RANKL $+25 \mathrm{ng} / \mathrm{ml}$ MCSF +100 nM sCT. $* P<0.05$ versus $O C$; $* * P<0.05$ versus $s C T$. No statistically significant difference was observed for antibody versus conjugate and antibody versus calcitonin.

associated with differing classes of bisphosphonates, the most commonly prescribed medication for osteoporosis (23). Estrogen hormone replacement therapy stimulates uterine and breast tissue, which predisposes the patient to neoplastic disease (24). Leg muscle cramps, joint aches and deep vein thrombosis has been associated with selective estrogen receptor modulator therapy (25). Similarly, conventional salmon calcitonin has shown limitations in antiresorptive efficacy, likely in part because of poor bioavailability to bone cells and the undesired uptake of

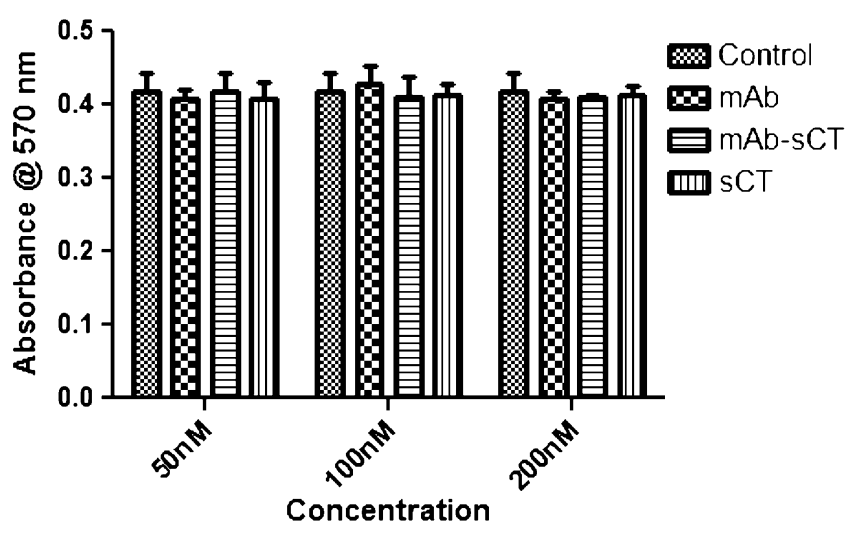

Fig. 9 In vitro cytotoxicity of mAb, mAb-sCT \& sCT on RAW 264.7 cells was determined by MTT assay. No demonstrable cytotoxicity was seen as measured by the absorbance of formazan solution formed after $4 \mathrm{~h}$ incubation with compounds, compared to that seen with untreated media. Samples were not statistically significant compared to the control at all concentrations.

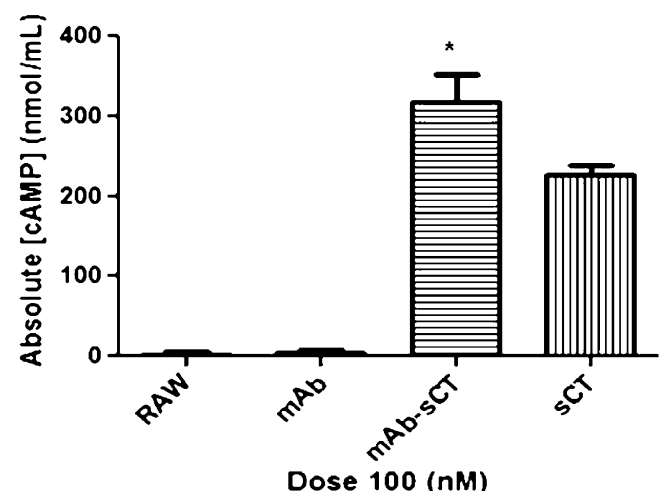

Fig. 10 Calcitonin receptor binding affinity and in vitro bioactivity of $m A b-$ sCT determined by using intracellular cAMP stimulation in human T47D cells. 50,000 cells/well were cultured for 2 days in 48-well plates in RPMI 1,640 containing insulin, and the phosphodiasterase activity was blocked using 3-IBMX. Cells were then treated with $100 \mathrm{nM}$ of mAb, mAb-sCT conjugate and $\mathrm{s} C T$, and the generated CAMP was assayed by CAMP ELISA. $* P<0.05$ versus all other cultures.

the drug by calcitonin receptors present in tissues other than bone $(26,27)$.

Since the pharmacological arrest of the osteoclast is the mainstay of treating systemic bone loss, drug delivery strategies that target the osteoclast directly would provide a potential therapeutic advantage at reducing systemic bone loss by selectively delivering an antiresorptive drug "cargo" to the osteoclast. Antibodies are renowned for their exquisite specificity of target recognition and generate highly selective outcomes following their systemic administration. As the RANK receptor is predominantly expressed on the surface of mature osteoclasts, we hypothesized that an anti-RANK antibody would facilitate an effective osteoclast targeting platform for the delivery of antiresorptive drug directly to the resorbing bone cells.

Salmon calcitonin, a single chain polypeptide hormone consisting of 32 amino acids, was chosen as a "model" drug for this study, as sCT therapy is known to inhibit and/or slow osteoclast-mediated resorptive bone loss, whilst positively influencing osteoinduction and bone formation (28). Hence, we designed a conjugative strategy whereby calcitonin would be coupled to an antibody directed predominantly against osteoclast-specific receptors, in order to impart osteoclast specificity to the attached calcitonin "cargo." Delivery of sCT to the osteoclast cells by virtue of an antibody-mediated targeting platform would ensure that the calcitonin drug remained primarily localized to bone tissue after systemic administration. This would directly contrast with the passive interaction of calcitonin with other tissues that express the calcitonin receptor, such as kidneys, liver, lungs, spleen, heart and thyroid. Hence, a delivery system capable of improving sCT targeting and localization to osteoclast could have a potential to positively impact 
sCT therapy, whilst reducing the drug concentration in non-bone loci containing the calcitonin receptors.

Using hybridoma technology, we generated and characterized a specific monoclonal antibody against recombinant human RANK receptor, and chemically coupled synthetic sCT to that anti-RANK mAb carrier. We further PEGylated sCT in the process of conjugation by using the polymer polyethylene glycol, which is known to improve the major disadvantages of conventional sCT by increasing solubility, stability, and efficacy and reducing the immunogenecity. It has also been established that PEGylation enhances the enzymatic stability of calcitonin (29) against proteolysis, by forming an effective shield against degradative enzymes (30) whilst improving its efficacy in viwo by increasing molecular size, thus contributing to reduced renal filtration (31) allowing less frequent administration (32).

The synthesized bio-conjugate was initially characterized using SDS-PAGE analysis and ELISA. Protein band above $150 \mathrm{kDa}$ was observed for the conjugate under nonreducing condition of SDS-PAGE analysis, and some bands were seen above $50 \mathrm{kDa}$ and $25 \mathrm{kDa}$ under reducing condition, thus suggesting that conjugation of PEGylated sCT with mAb had occurred. An ELISA further verified the successful conjugation of sCT to our mAb, as the anticalcitonin secondary antibody reagent would only detect immobilized calcitonin residues that were part of the primary anti-RANK conjugates that were binding to the RANK-coated wells of the ELISA plate. A slight signal was observed for PEGylated SCT, and we assume that this could be due to the reaction of thiol reactive MAL functional group of PEGylated sCT with the free thiol group in BSA used as a blocking agent. In the case of conjugate, such MAL groups were utilized in the reaction with free thiol groups generated in antibody.

In order to verify the efficacy of mAb-sCT conjugates, we employed in vitro osteoclast-like cell cultures. It is well established that osteoclasts are derived from cells of the monocyte/macrophage lineage and are formed after the cellular fusion of their mononuclear precursors (33). Hence, we utilized the RAW 264.7 cell line that can be differentiated in vitro into osteoclast-like cells after exposure to M-CSF and RANKL cytokines. RANKL is the essential factor necessary for osteoclast differentiation, whilst M-CSF (also known as C.SF-1) has also been identified as an essential factor necessary to initiate osteoclastogenesis (34). The main role of M-CSF is to act as a survival factor for osteoclast precursor cells and to induce the expression of the RANK receptor for stimulation by the RANKL.

Immunocytochemistry confirmed the osteoclast targeting potential of our generated anti-RANK $\mathrm{mAb}$ in vitro. Fluorescent staining of our anti-RANK mAb was specifi- cally localized to cellular surface receptors. DAPI staining showed the successful generation of multinucleated osteoclast-like cells. No demonstrable FITC staining was seen for negative controls. These results support the evidence that osteoclast-like cells generated from RAW 264.7 precursor cells express the RANK receptor, and our generated anti-RANK antibody can be used as a potential osteoclast targeting platform. The successful generation of osteoclast-like cells was also confirmed by TRAP staining, which confirmed multinucleated cells with maroon-colored dye deposits due to the presence of TRAP, the enzyme that has been used as a marker of osteoclast function for more than 20 years (35).

To determine the in vitro efficacy of the anti-RANK mAb-sCT conjugate on osteoclast activity, we quantified osteoclast activity using a spectrophotometric total culture TRAP activity assay and a mineral resorption assay. TRAP has been shown to be a specific and sensitive indicator of bone resorption (36) and contributes to the intracellular processing of primary bone matrix degradation products prior to its final release through the basolateral membrane of resorbing osteoclast cells $(37,38)$. Osteoclasts, as the bone-resorbing cells of the body, further show the ability to dissolve the mineralized inorganic phase of bone matrix known as hydroxyapatite (39). We found that the use of our conjugate in culture significantly reduced TRAP enzyme activity as compared to sCT alone, and use of the $\mathrm{mAb}$ alone also showed inhibition of TRAP enzyme activity. Although the cause for this effect shown by our anti-RANK antibody alone has not been tested, we speculate that in binding the RANK receptor, the antibody may be functioning as a receptor antagonist. That competitive binding would interfere with the interaction of RANKL with RANK, an essential step of osteoclast differentiation. Nonetheless, further antiresorptive and/or anabolic effector functions could be attributed with the development of a targeted delivery strategy to osteoclast cell receptors. We further evaluated if the mAb-sCT conjugate had an effect on the ability of osteoclasts to resorb bone. We cultured osteoclast-like cells on calcium phosphate-coated culture plates in the presence of conjugate, antibody or sCT. We found that both the conjugate and the $\mathrm{mAb}$ alone were capable of significantly reducing the resorption of mineral layer as compared to the sCT alone. Then, we performed cyclic AMP assay to confirm that bioactivity of conjugated calcitonin. Human breast carcinoma T47D cells known to express the calcitonin receptor were used for this purpose. Binding of $\mathrm{sCT}$ with its receptor activates adenylyl cyclase, an enzyme responsible for the generation of cAMP. Our in vitro cAMP assay results confirmed that the modification of sCT by conjugation did not interfere with its ability to bind the calcitonin receptor and trigger its biological activity. We measured the 
conjugate to have increased activity over calcitonin alone, and the exact reason for that effect is still not clear. It is likely our conjugation strategy allowed one mole of antibody to become conjugated with greater than one mole of calcitonin. It has been reported that when $\operatorname{IgG}$ is treated with a 10 molar excess of Traut's reagent, 3-7 thiol groups are generated, which may result in the attachment of 3-7 PEGylated calcitonin molecules per molecule of $\mathrm{IgG}$.

The results obtained so far have been quite encouraging from the generation of antibody and to the synthesis and in vitro evaluation of the conjugate. The conjugate and the antibody alone showed good efficacy in TRAP activity inhibition and resorption pit assay. However, cAMP assay showed that the conjugate served the purpose of delivering active drug cargo. The conjugate showed the ability to bind the calcitonin receptors and generate intracellular cyclic AMP, thus showing an advantage over the antibody alone.

With the advent of novel RANKL-scavenging antibodybased antiresorptive strategies (such as Denosumab- Prolia ${ }$, Amgen Inc.), it may further prove efficacious to target the RANK receptor directly with an antibody, in order to successfully antagonize receptor signaling and osteoclast function. However, neither the "scavenging" nor "antagonist" motives of those antiresorptive strategies focus on the targeted delivery of a given drug cargo (such as our antiRANK mAb-conjugate design) which would function as a universal osteoclast targeting platform.

One potential limitation of our osteoclast targeting platform remains that RANKL-RANK signaling is not entirely restricted to osteoclasts and their precursors, but is known to be involved in the regulation of other cell types. Studies have that RANKL-RANK signaling controls the development of lactating mammary glands in pregnancy, with the highest levels being at day 15.5 in mice $(40,41)$. Several studies that looked at transgenic Rankl-/- and Rank-/- mice found that there was a complete absence of lymph nodes (42). This led to further studies involving patients with an osteoclast-poor form of the autosomal recessive osteopetrosis (ARO), where they found the cause of the disease to be various mutations in RANKL (43). The same research then found that these patients showed no palpable signs of lymph nodes, further strengthening the theory that RANKL-RANK signaling is involved in lymph node formation in humans. RANK is further known to be expressed by monocyte/macrophages and dendritic cells, specialized immune cells programmed to capture and process antigens in the body and present them to naïve T-cells (44). However, the targeting of drug "cargo" to the RANK receptors of those cells may not necessarily result in drug action with those cells if the cargo is carefully chosen not to affect those cell types. For example, it is highly unlikely for any cell (other than the mature osteoclast) to express both the RANK receptor and calcitonin receptor at the same time. Thus, the Boolean "and/or" logic can be applied to the selection of the differential cargo targeting to cellular phenotypes expressing the RANK receptor at any given time. The extent of this limitation remains to be fully determined, however, should not necessarily preclude the further exploration of RANK receptor targeting strategies, which may in turn provide a more comprehensive understanding of RANK signaling and crosstalk with other signaling pathways.

\section{CONCLUSION}

This study details the generation of an antibody-mediated osteoclast-targeting platform. This drug-delivery strategy may find utility as "a universal osteoclast-targeting platform" in order to directly target and deliver antiresorptive agents, anti-inflammatory agents, cathepsin $\mathrm{K}$ inhibitors, disintegrins, $\mathrm{H}^{+}$-ATPase inhibitors, and so on, directly to osteoclast cells. We have shown this platform is capable of being employed as an antiresorptive strategy, and our efforts will now center on the in vivo evaluation of this targeting strategy.

\section{ACKNOWLEDGMENTS}

This paper was presented in-part as a poster at the 13th Annual Canadian Society for Pharmaceutical Sciences (CSPS) Symposium, Richmond BC, June 2-5, 2010. This research was funded by the Osteoarthritis (OA) Alberta Team Grant from the Alberta Heritage Foundation for Medical Research (AHFMR).

Open Access This article is distributed under the terms of the Creative Commons Attribution Noncommercial License which permits any noncommercial use, distribution, and reproduction in any medium, provided the original author(s) and source are credited.

\section{REFERENCES}

1. Roodman G. Advances in bone biology - the osteoclast. Endocr Rev. 1996;17:308-32.

2. Riggs BL, Khosla S, Melton LJ. Sex steroids and the construction and conservation of the adult skeleton. Endocr Rev. 2002;23:279302.

3. Manolagas SC. Birth and death of bone cells: basic regulatory mechanisms and implications for the pathogenesis and treatment of osteoporosis. Endocr Rev. 2000;21:115-37.

4. Pelletier JP, Boileau C, Brunet J, Boily M, Lajeunesse D, Reboul $\mathrm{P}$, et al. The inhibition of subchondral bone resorption in the early 
phase of experimental dog osteoarthritis by licofelone is associated with a reduction in the synthesis of MMP-13 and cathepsin K. Bone. 2004;34:527-38.

5. Hirayama T, Danks L, Sabokbar A, Athanasou NA. Osteoclast formation and activity in the pathogenesis of osteoporosis in rheumatoid arthritis. Rheumatology. 2002;41:1232-9.

6. Nakagawa N, Kinosaki M, Yamaguchi K, Shima N, Yasuda H, Yano $\mathrm{K}$, et al. RANK is the essential signaling receptor for osteoclast differentiation factor in osteoclastogenesis. Biochem Biophys Res Commun. 1998;253(2):395-400.

7. Boyle WJ, Simonet WS. Lacey DL Osteoclast differentiation and activation. Nature. 2003;423:337-42.

8. Faccio R, Takeshita S, Zallone A, Ross FP, Teitelbaum SL. c-Fms and the alphavbeta3 integrin collaborate during osteoclast differentiation. J Clin Invest. 2003;111:749-58.

9. Zaidi M, Inzerillo AM, Moonga BS, Bevis PJ, Huang CL. Bone. 2002;30:655-63.

10. Breimer LH, Macintyre I, Zaidi M. Peptides from the calcitonin genes: molecular genetics, structure and function. Biochem J. 1988;255:377-90.

11. Moonga BS, Moss DW, Patchell A, Zaidi M. Intracellular regulation of enzyme secretion from rat osteoclasts and evidence for a functional role in bone resorption. J Physiol. 1990;429(1):2945

12. Mancini L, Moradi-Bidhendi N, Brandi ML, Perretti M, MacIntyre I. Modulation of the effects of osteoprotegerin (OPG) ligand in a human leukemic cell line by OPG and calcitonin. Biochem Biophys Res Commun. 2000;279:391-7.

13. Shahhosseini S, Das D, Qiu X, Feldmann H, Jones SM, Suresh MR. Production and characterization of monoclonal antibodies against different epitopes of Ebola virus antigens. J Virol Methods. 2007;143(1):29-37.

14. Kammila S, Das D, Bhatnagar PK, Sunwoo HH, ZayasZamora G, King M, et al. A rapid point of care immunoswab assay for SARS-CoV detection. J Virol Methods. 2008;152 (1-2):77-84.

15. Köhler G, Milstein C. Continuous cultures of fused cells secreting antibody of predefined specificity. Nature. 1975;256:495-7.

16. Bu R, Borysenko CW, Li Y, Cao L, Sabokbar A, Blair HC. Expression and function of TNF-family proteins and receptors in human osteoblasts. Bone. 2003;33(5):760-70.

17. Traut RR. Methyl 4-mercaptobutyrimidate as a cleavable crosslinking reagent and its application to the Escherichia coli $30 \mathrm{~S}$ ribosome. Biochemical. 1973;12(17):3266-73.

18. Jue R. Addition of sulfhydryl groups to Escherichia coli ribosomes by protein modification with 2-iminothiolane (methyl 4-mercaptobutyrimidate). Biochemical. 1978;17 (25):5399-405.

19. Ellman GL. Tissue sulfhydryl groups. Arch Biochem Biophys. 1959;82(1):70-7.

20. Stevenson CL, Tan MM. Solution stability of salmon calcitonin at high concentration for delivery in an implantable system. J Pept Res. 2000;55:129-39.

21. Rahman MM, Bhattacharya A, Fernandes G. Conjugated linoleic acid inhibits osteoclast differentiation of RAW264.7 cells by modulating RANKL signaling. J Lipid Res. 2006;47 (8):1739-48.

22. Bhandari KH, Newa M, Uludag H, Doschak MR. Synthesis, characterization and in vitro evaluation of a bone targeting delivery system for salmon Calcitonin. Int J Pharm. 2010;394 (1-2):26-34.

23. de Groen PC, Lubbe DF, Hirsch LJ, Daifotis A, Stephenson W, Freedholm D, et al. Esophagitis associated with the use of alendronate. N Engl J Med. 1996;335:1016-21.

24. Rossouw JE, Anderson GL, Prentice RL, LaCroix AZ, Kooperberg C, Stefanick ML, et al. Risks and benefits of estrogen plus progestin in healthy postmenopausal women: principal results from the Women's Health Initiative randomized controlled trial. JAMA. 2002;288(3):321-33.

25. de Villiers TJ. Clinical issues regarding cardiovascular disease and selective estrogen receptor modulators in postmenopausal women. Climacteric. 2009;12 Suppl 1:108-11.

26. Huwyler R, Born W, Ohnhaus EE, Fischer JA. Plasma kinetics and urinary excretion of exogenous human and salmon calcitonin in man. Am J Physiol Gastrointest Liver Physiol. 1979;236:G159.

27. Fouchereau-Peron M, Moukhtar MS, Benson AA, Milhaud G. Characterization of specific receptors for calcitonin in porcine lung. Proc Natl Acad Sci USA. 1981;78:3973-5.

28. Okubo Y, Bessho K, Fujimura K, Kusumoto K, Ogawa Y, Iizuka T. Effect of e-calcitonin on osteoinduction by recombinant human bone morphogenetic perotein-2. Biochem Biophys Res Commun. 2000;269:317-21.

29. Lee KG, Moon SG, Park MO, Lee JT, Na DH, Yoo SD, et al. Isolation, characterization, and stability of positional isomers of mono-PEGylated salmon calcitonins. Pharm Res. 1999;16:813-8.

30. Cleland JL, Daugherty A, Mrsny R. Emerging protein delivery methods. Curr Opin Biotechnol. 2001;12:212-9.

31. Fee CJ. Size comparison between proteins PEGylated with branched and linear poly(ethylene glycol) molecules. Biotech Bioeng. 2007;98:725-31.

32. Hamidi M, Azadi A, Rafiei P. Pharmacokinetic consequences of PEGylation. Drug Deliv. 2006;13:399-409.

33. Vaananen HK, Laitala-Leinonen T. Osteoclast lineage and function. Arch Biochem Biophys. 2008;473:132-8.

34. Yoshida H, Hayashi S, Kunisada T. The murine mutation osteopetrosis is in the coding region of the macrophage colony stimulating factor gene. Nature. 1990;345:442-4.

35. Minkin C. Bone acid phosphatase: tartrate-resistant acid phosphatase as a marker of osteoclast function. Calcif Tissue Int. 1982;34:285-90.

36. Halleen JM, Ranta R. Tartrate-resistant acid phosphatase as a serum marker of bone-resorption. Am Clin Lab. 2001;20:2930.

37. Vaananen HK, Zhao H, Mulari M, Halleen JM. The cell biology of osteoclast function. J Cell Sci. 2000;113:377-81.

38. Vaaraniemi J, Halleen JM, Kaarlonen K, Ylipahkala H, Alatalo SL, Andersson G. Intracellular machinery for matrix degradation in bone-resorbing osteoclasts. J Bone Miner Res. 2004;19:143240.

39. Teitelbaum SL. Osteoclasts: what do they do and how do they do it? Am J Pathol. 2007;170:427-35.

40. Fata JE, Kong YY, Li J, Sasaki T, Irie-Sasaki J, Moorehead $\mathrm{RA}$, et al. The osteoclast differentiation factor osteoprotegerinligand is essential for mammary gland development. Cell. 2000;103 (1):41-50

41. Gonzalez-Suarez E, Branstetter D, Armstrong A, Dinh H, Blumberg H. C.RANK Overexpression in transgenic mice with mouse mammary tumor virus promoter-controlled RANK increases proliferation and impairs alveolar differentiation in the mammary epithelia and disrupts lumen formation in cultured epithelial acini. Mol Cell Biol. 2007;27:1442-54.

42. Dougall WC, Glaccum M, Charrier K, Rohrbach K, Brasel K, Smedt TD, et al. RANK is essential for osteoclast and lymph node development. Genes Dev. 1999;13:2412-24.

43. Sobacchi C, Frattini A, Guerrini MM, Abinun M, Pangrazio A, Susani L, et al. Villa A\& Helfrich MH.Osteoclast-poor human osteopetrosis due to mutations in the gene encoding RANKL. Nat Genet. 2007;39:960-2.

44. Ferrari-Lacraz S, Ferrari S. Do RANKL inhibitors (denosumab) affect inflammation and immunity? Osteoporos Int. 2010. 